\title{
Polarization-Independent Electrically Tunable Holographic Polymer Dispersed Liquid Crystals Grating Doped with Chiral Molecules
}

\author{
Hui LI ${ }^{1,2,4}$, Fan PAN ${ }^{3}$, Yuntao WU ${ }^{1,2}$, Yanduo ZHANG ${ }^{1,2}$, Xiaolin XIE $^{4}$ \\ ${ }^{1}$ School of Computer Science and Engineering, Wuhan Institute of Technology, Wuhan 40073, P. R. China \\ ${ }^{2}$ Hubei Key Laboratory of Intelligent Robot, Wuhan 40073, P. R. China \\ ${ }^{3}$ College of Post and Telecommunication, Wuhan Institute of Technology, Wuhan 40073, P. R. China \\ ${ }^{4}$ School of Chemistry and Chemical Engineering, Huazhong University of Science and Technology, Wuhan 40073, P. R. \\ China
}

crossref http://dx.doi.org/10.5755/j01.m.s.23.2.16312

Received 30 September 2016; accepted 04 November 2016

\begin{abstract}
This study proposes a holographic grating made of polymer dispersed liquid crystal (PDLC), with a small amount of chiral molecules doped into PDLC material. The major advantage of this grating is that it is independent of light polarization. This characteristic was verified by applying the interference beam intensity of a He-Cd laser at $150 \mathrm{~mW} / \mathrm{cm}^{2}$, with an incidence angle between the two interference beams of $24^{\circ}$, for an irradiation curing duration of $120 \mathrm{~s}$. The observed periodic structure of the grating is consistent with the theoretical value. As chiral molecules are doped, nematic-LC experiences a phase-change in the grating. However, the electro-optical features are only slightly affected. This proposed grating has greatly potential in 3D imaging because of its polarization-independent feature. Keywords: chiral molecules dopant, polymer dispersed liquid crystals, grating, polarization-independent.
\end{abstract}

\section{INTRODUCTION}

With the rapid development of technology, grating has become an indispensable optical instrument with applications in many fields, such as integrated optics, optical holography, spectral analysis, data storage, optical switching, and image processing. Various types of diffraction gratings have attracted attention from researchers, including diffraction grating, orthogonal grating, holographic grating, and blaze grating [1-4]. Among these types, holographic grating has become an especially exciting research topic because of its potential uses in 3D displays. In the field of material technologies, polymer dispersed liquid crystal (PDLC) represents a new kind of electro-optical material with uses in fabricating electro-optical devices, especially holographic grating $[5,6]$. Generally, holographic-PDLC (H-PDLC) grating operates according to the optical coherent superposition principle. H-PDLC grating has been widely utilized for many purposes, such as eliminating aberrations related to atmospheric disturbances, optical encryption, measuring optical inhomogeneity and holography with stress, studying transient phenomenon, storing information, and displaying images in 3D movies [7-9].

Researchers from several different countries have proposed fabrication methods intended to produce $\mathrm{H}$ PDLC grating $[10,11]$. However, only a few studies have examined the solution to the polarization-independent problem of H-PDLC grating. This issue is mainly caused by nematic liquid crystal (LC), with the optical anisotropy of nematic-LC constituting the key factor. Methods proposed to overcome this problem include changing the

\footnotetext{
* Corresponding author. Tel.: +86-27-87992007; fax: +86-27-87992007 E-mail address: lihui00317@163.com (H. Li)
}

structure's design and photo-alignment with dopant UV sensitive monomers, among others [12,13]. However, these methods are generally complicated. This paper proposes a relatively simple method to solve this problem, by doping a small amount of chiral molecules into PDLC material.

Section 2 of this paper presents the fabrication workflow of the proposed H-PDLC grating. Section 3 describes experimental results that prove its polarizationindependent feature and discuss its other classic electrooptical features. Finally, Section 4 describes conclusions about the proposed H-PDLC grating doped with chiral molecules.

\section{EXPERIMENTAL PROCEDURE}

H-PDLC grating primarily consists of an LC/monomer mixture doped with chiral molecules and two indium tin oxide (ITO) substrates. The H-PDLC grating monomers used in this study are 2-Ethylhexyl acrylate (EHA, purity $>99 \%$, from Acros Oganics Co.), Ethylene dimethacrylate (EDMA, purity $>98 \%$, from Acros Oganics Co.), N-vinyl pyrrolidone (NVP, purity $>99 \%$, from Aldrich Co.), and Pentaerythritol Tetraacrylate (PETTA, from Aldrich Co.). Other important materials utilized include nematic-LC (P0616A, from Shijiazhuang Chengzhi Yonghua Display Materials Co., as shown in Fig. 1 a), Irgacure 784 (purity $>99 \%$, from Jingmen Yukui Huagong Co., as shown in Fig. 1 b), Benzoyl peroxide (BPO, from Sinopharm Chemical Reagent Co.), and chiral dopant (ZLI-811, from Merck Co., as shown in Fig. 1 c). These chemical agents were all directly utilized without any further processing, except for BPO, which was purified by recrystallizing from petroleum ether solution. The processed $\mathrm{BPO}$ was dried in a vacuum at $35{ }^{\circ} \mathrm{C}$ for 24 hours. 

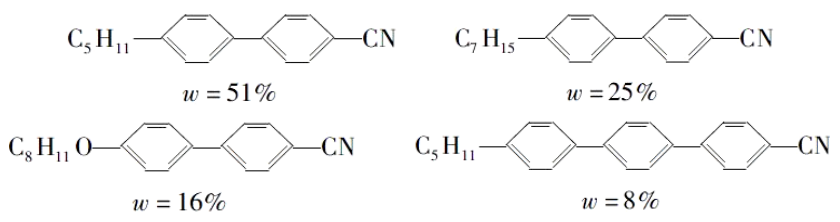

a

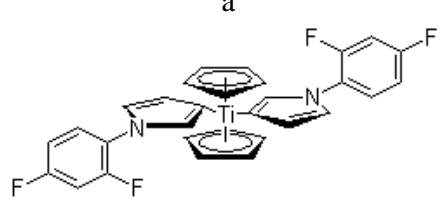

b

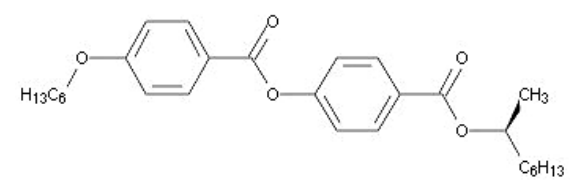

$\mathrm{c}$

Fig. 1. Chemical structure: a-structure of nematic-LC P0616A; $\mathrm{b}$ - structure of Irgacure 784; c - structure of ZLI-811

The most important step in fabricating H-PDLC grating is acquiring the PDLC film. This study adopted the following technological process. At first, the nematic-LC host and doped chiral molecules were mixed at ratios of $\mathrm{X}$ : $100-\mathrm{X}$ wt.\% with a precision electronic scale, where $\mathrm{X}$ was $0,5,10$, and 20 . The mixture was poured into a bottle and slightly heated on a heating plate. A gyrotron shaker was applied for five minutes, as was an ultrasonication. This resulted in a uniform mixture of the nematic-LC and chiral molecules. The materials in the next stage of the process were the monomers EHA, NVP, EDMA, PETTA as well as nematic-LC P0616A (doped with chiral molecules). The optimized weight ratios of those materials were 6.8:2.2:1.0:4.4:7.5. The above proportion value is the mass fraction ratio between those mentioned chemical reagents. The two shakers mentioned above were utilized for five minutes each, until the nematic-LC, monomers and chiral molecules were uniformly mixed. In addition, Irgacure 784 (about 1.8 wt.\%) and BPO (about 2.2 wt.\%) were continuously stirred at room temperature for 12 hours. In order to avoid reactions with light, the preceding steps were all conducted in a darkroom. Then, two ITO glasses sandwiched with $10 \mu \mathrm{m}$ spacers were utilized to fabricate an empty LC cell. The prepared solution was poured into this empty LC cell through capillary action. Fig. 2 shows the experiment setup for fabricating the H-PDLC grating with holographic technology. Two laser beams were then applied. The first was a He-Cd laser beam, $442 \mathrm{~nm}$ wavelength, $1 \sim 150 \mathrm{~mW} / \mathrm{cm}^{2}$ power, TEM00 mode, and $\mathrm{CW}$ type, as a holographic interfering beam source. A beam splitter of $150 \mathrm{~mW} / \mathrm{cm}^{2}$ was utilized to divide this laser beam into two interference beams with the same intensity, between which was an incidence angle of $24^{\circ}$. The curing duration of irradiation was $120 \mathrm{~s}$. Generally, holographic-PDLC (HPDLC) grating operates according to the optical coherent superposition principle. Based on equation:

$\Lambda=\lambda / 2 \sin \theta / 2$, where $\Lambda$ is the theoretical grating period, $\lambda$ is the incident laser beam wavelength and $\theta$ is the incidence angle between two interference beams, the theoretical grating period of the grating is $1 \mu \mathrm{m}$. The second laser beam was a $\mathrm{He}-\mathrm{Ne}$ laser, used as a probe beam. After curing irradiation, both phase-change and phase-separation occurred in the LC cell. Ultimately, this process produced transmission H-PDLC grating.

As described above, the He-Ne laser functioned as a probe light source. The intensities of the zero-order and first-order diffraction transmission lights were recorded by a photodiode detector set at $20 \mathrm{~cm}$ away from the sample. The recorded data were then analyzed by a computer. Applied voltage tunable from $0 \mathrm{~V}$ to $130 \mathrm{~V}$ at $1 \mathrm{KHz}$ was loaded at both ITO electrodes of the H-PDLC grating. The operation voltage was used just during measuring characterization of the proposed grating, which were not utilized during the fabricating procedure.

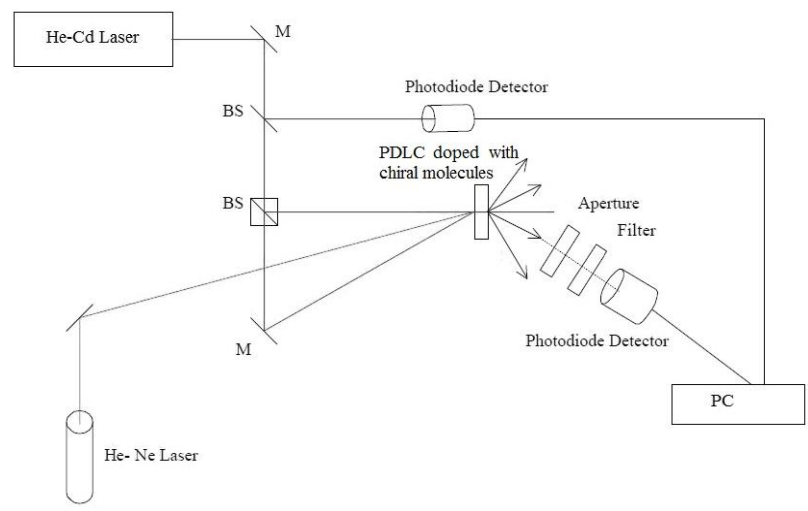

Fig. 2. Experimental setup for fabricating H-PDLC grating

\section{RESULTS AND DISCUSSION}

This paper describes some experiments in detail in order to demonstrate the notable characteristics of the proposed H-PDLC grating doped with chiral molecules. This section first describes the structure of the grating, before discussing its major advantage.

Once the materials were cured, an internal image of the grating was taken by HR-FESEM (Sirion 200, Netherlands), as shown in Fig. 3. After phase-separation, LC-rich and polymer-rich areas formed in the grating. In order to obtain the best results, the sample was cut into a small piece. After immersion in an $\mathrm{n}$-hexane solution for 2 days, the most of LCs in the sample could be removed by the above method. The whole measurement of removing LCs was in a dry condition at room temperature to avoid effect of humidity and temperature factors. After the sample was sprayed by some platinum, the FESEM image could be clearly observed. The periodic structure was measured at $1 \mu \mathrm{m}$ width, exactly the same as the theoretical value, as shown in Fig. 3. This indicates that the phaseseparation between LCs and polymers occurred as scheduled. In Fig. 3, the minimum scale of SEM is just $1 \mu \mathrm{m}$. The narrow areas in Fig. 3 are mostly in the LC phase, while the wide areas mostly belong to the polymer phase. Based on holographic technology theory, the interference beam is the major factor in forming this structure [14]. When the LC/monomer mixture was 
exposed to interference beam, the phase-separation occurred. As the phase-separation between LCs and polymers is a sophisticated phenomenon, there are so many factors to affect the final result. Thus, brief analysis was presented in this study, and the specific process proceeded as follows [14]. In regions with strong light intensity, monomers solidified into polymers, meaning that some monomers were consumed and the chemical potential of monomers in those areas was reduced. Therefore, in order to satisfy thermodynamic equilibrium, monomers in areas with weak light intensity diffused into areas with strong light intensity, which partly supplemented the chemical potential of the other monomers. Correspondingly, in regions with strong light intensity, the chemical potential of LCs increased after the consumption of some monomers. Similarly, in order to satisfy thermodynamic equilibrium, the LCs in areas with strong light diffused into areas with weak light intensity. In this way, the polymer-rich region formed where the light intensity was strong, and the LC-rich region formed where the light intensity was weak. Because of the speed of polymerization, some LC molecules became wrapped in polymers. As a result, the LC-phase was quite small, while the polymer-phase was relatively large. In other words, the intensity of beam was the most important factor to the polymerization speed. Therefore, the light intensity was different, and the speed of the polymerization was also different. In order to verify this process, statistics measurement was established to evaluate the number of LCs in the grating. Based on measurements from a digital micrograph (Gatan Inc.), the average diameter of LC droplets in the grating is about $87 \pm 25 \mathrm{~nm}$, and the total number of LC droplets, 161, could be got by using the digital micrograph. The size of LCs in phase-separation represents an essential factor for estimating degree of phase-separation. And the bigger LC droplet's diameter, the better phase-separation. These results therefore indicate that phase-separation took place in the grating.

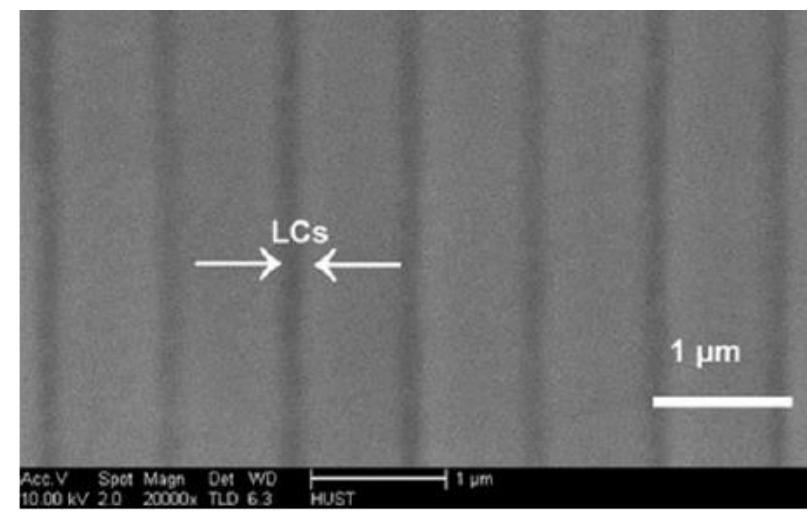

Fig. 3. FESEM image of the proposed transmission H-PDLC grating, where the scale of SEM is just $1 \mu \mathrm{m}$ the same as the grating period

To verify the polarization-independent feature of the grating, the intensities of transmission lights under different applied voltages were measured at zero-order and first-order diffraction points by still using the experimental setup as shown in Fig. 2. These measurements revealed information about different concentrations of doped chiral molecules, as shown in Fig. 4. Those measurement data were got by many times measuring. Because the grating is a LC device, the transmission light intensity can be altered based on the external applied voltages. Compared with the tested images, the transmission light intensities varied between p-polarized and s-polarized light at $0 \mathrm{wt} \%$ and 5 wt.\% doped chiral molecules, indicating that the LCs in the grating remained nematic-LC without experiencing a phase-change. This is because the concentration of doped chiral molecules was not high enough for phase-change to occur in the grating. With the dopant of those chiral molecules, a periodic helical structure was formed in nematic-LC because of helix twisting force. As phasechange occurred, the nematic-phase was changed to cholesteric-phase. At this concentration of dopant the proposed grating, like other H-PDLC gratings, experienced a problem of polarization, which is dependent on the polarization direction of the incident light. Under these conditions, the refractive index of extraordinary light of nematic-LC varies based on the external applied voltages. However, the refractive index of ordinary light of nematicLC does not change. Because of these features, the transmission light intensity in the p-polarized direction changes considerably, while the transmission light intensity in the s-polarized direction shows nearly no variations. The experimental results support this hypothesis. However, when the concentrations of doped chiral molecules rise to $10 \mathrm{wt} \%$ or $20 \mathrm{wt} . \%$, the transmission light intensity becomes identical between spolarized and p-polarized, in contrast to the comparison at low concentrations. This implies that the changing trends are the same at both zero-order and first-order diffraction points, because the concentration of the doped chiral molecules exceeds a threshold value. Under these conditions, the nematic-LC in the H-PDLC grating experiences a phase-change in which it is converted to cholestic-LC. As has been established, cholestic-LC has a spiral structure with a polarization-independent feature. As a result, the dopant chiral molecules represent a critical factor for realizing polarization-independent grating. Based on the images in Fig. 4, different concentrations of doped chiral molecules create an obvious contrast. Relatively speaking, the $10 \%$ concentration of chiral molecules is the best value. Based on this relatively simple method, nematic-LC in the grating is converted to cholesteric-LC. The concentration of doped chiral molecules is low so that the single twist structure was formed. And internal structure can determine feature of LC. In this way, LC doped with chiral molecules have two more optical features, optical active and dichroism [15]. Those features directly relate to polarization feature of LC. As the cholesteric-LC is dominant, the alternations of the refractive index of LCs different from those of nematicLC. In principle, when the external applied voltage is altered, the pitch of the cholesteric-LC changes. Therefore, the refractive index of cholesteric-LC can be adjusted by external applied voltage. Fig. 4 shows results show that are consistent with predictions, suggesting that the external applied voltage can be applied to adjust the grating properties. Moreover, the proposed grating has a polarization-independent feature, which can be tunable using an external applied voltage.

Diffraction efficiency of the grating represents a third 
important feature for the H-PDLC grating.

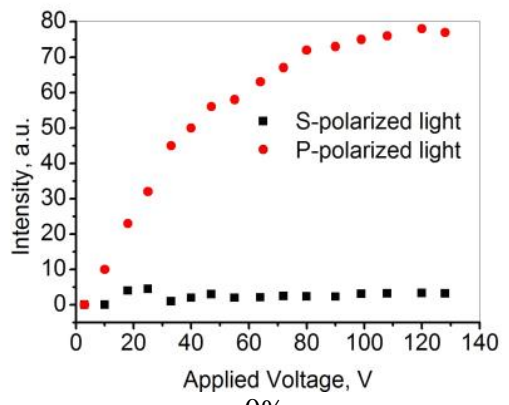

$0 \%$
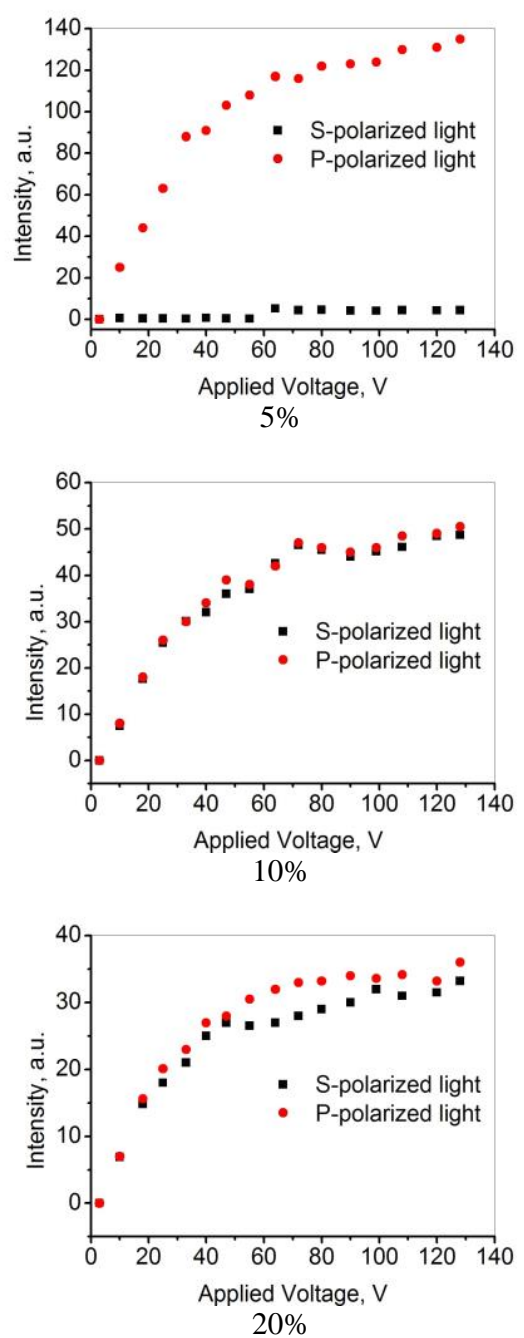

Fig. 4. a - transmission light intensities of H-PDLC grating doped with different concentrations of chiral dopant at zeroorder diffraction point

Using the same experimental setup in Fig. 2, the photodiode detector was replaced by a CCD camera (3 Mega-pixel, 1/2 inch), which detected the recorded image. Fig. 5 shows the one dimensional diffraction image by the CCD camera. The image shows five diffraction points. The middle one is the zero-order diffraction point, and the left and right ones are the first-order diffraction points. The second-order diffraction points are visible but not very clear at the edge of the image.

Table 1 displays the diffraction efficiencies of the proposed grating under different concentrations of doped chiral molecules, based on the equation

$\eta=I_{d} /\left(I_{d}+I_{t}\right)$,

where $\eta$ is diffraction efficiency, $I_{d}$ is diffraction light intensity, and $I_{t}$ is transmission light intensity. The external applied voltage ranges from $0 \mathrm{~V}$ to $130 \mathrm{~V}$ at $1 \mathrm{KHz}$.

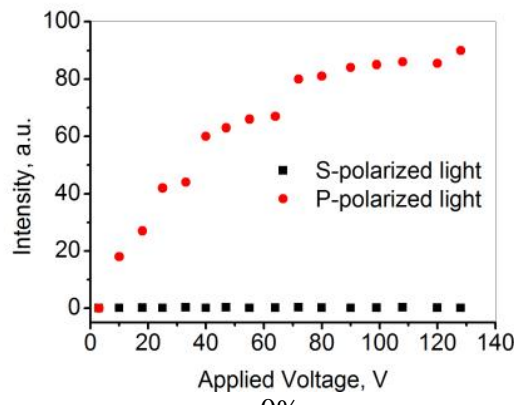

$0 \%$

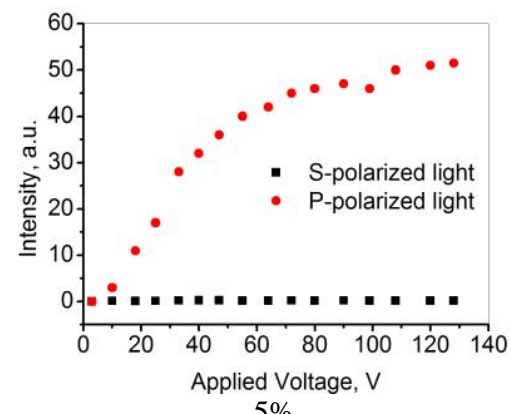

$5 \%$
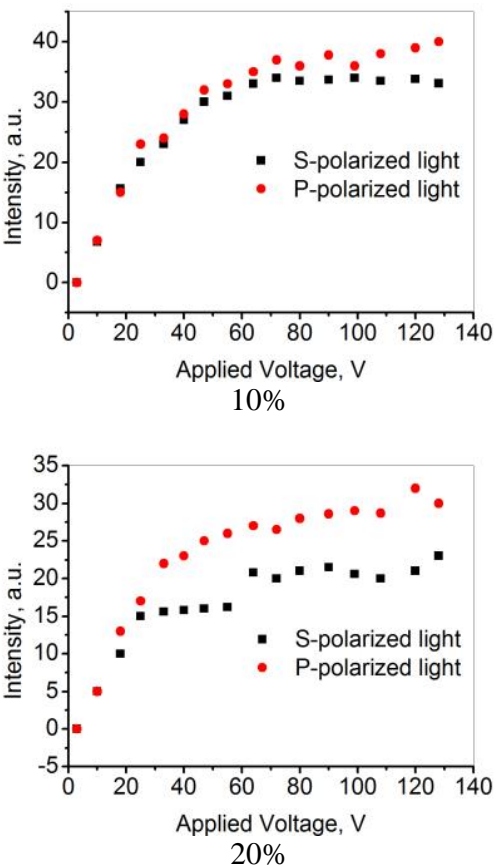

Fig. 4. $\mathrm{b}$ - transmission light intensities of H-PDLC grating doped with different concentrations of chiral dopant at first-order diffraction point

Table 1 indicates that there are no obviously changes between different concentrations of doped chiral molecules. This proves that the doped chiral molecules don't influence the electro-optical features of the proposed grating. In conclusion, the classic electro-optical features 
of H-PDLC grating do not vary after doping with chiral molecules.

\section{6}

Fig. 5. One dimensional diffraction image by CCD camera

Table 1. Diffraction efficiencies of the proposed H-PDLC grating under different concentrations of doped chiral molecules

\begin{tabular}{|c|c|c|c|c|c|c|c|}
\hline Concentrations & $0 \mathrm{~V}$ & $20 \mathrm{~V}$ & $40 \mathrm{~V}$ & $60 \mathrm{~V}$ & $80 \mathrm{~V}$ & $100 \mathrm{~V}$ & $120 \mathrm{~V}$ \\
\hline $0 \%$ & 39.8 & 39.5 & 39.1 & 39.0 & 38.9 & 38.8 & 38.7 \\
\hline $5 \%$ & 40.3 & 40.2 & 39.8 & 39.7 & 39.6 & 39.5 & 39.3 \\
\hline $10 \%$ & 39.7 & 39.6 & 39.2 & 39.1 & 39.0 & 38.9 & 38.8 \\
\hline $20 \%$ & 39.8 & 39.6 & 39.1 & 39.0 & 39.0 & 38.9 & 38.8 \\
\hline
\end{tabular}

\section{CONCLUSIONS}

This study demonstrates a relatively simple method for producing electrical tunable H-PDLC grating. First, different concentrations of chiral molecules were doped into PDLC material. Based on phase-change theory, nematic-LC was converted into the cholesteric phase. These conditions effectively eliminated the polarization effect of the H-PDLC grating, and the feature of polarization-independent was verified experimentally. The most important feature of the proposed H-PDLC grating is that it has the same diffraction efficiency in both polarized directions. This study's results also suggest that the classic electro-optical features of this H-PDLC grating do not change after doping with chiral molecules in this study. The proposed H-PDLC grating has greatly potential applications in 3D display.

\section{Acknowledgments}

This work was supported by National Natural Science Foundation of China (61671337), a grant from the Research Foundation of Education Bureau of Hubei Province China (B2016489), a grant from the Natural Science Foundation of Hubei Province of China (2014CFB771), China Postdoctoral Science Foundation (2014M562017), the Science Foundation of the Wuhan Institute of Technology (Q201601), and the Open Foundation for Hubei Key Laboratory of Intelligent Robot (HBIR 201605).

\section{REFERENCES}

1. Wang, J.H., Zhang, W., Cui, Y.W., Teng, S.Y. Fresnel Diffraction of Fractal Grating and Self-Imaging Effect Applied Optics 53 (10) 2014: pp. 2105-2111. https://doi.org/10.1364/AO.53.002105

2. Liang, Y., Mi, L.N., Peter, R.H. Femtosecond Laser Writing of Phase-Tuned Volume Gratings for Symmetry Control in 3D Photonic Crystal Holographic Lithography Optical Materials Express 5 (3) 2015: pp. 515-529.
https://doi.org/10.1364/OME.5.000515

3. Kakauridze, G.A., Kilosanidze, B.N. PolarizationHolographic Gratings that Form Plane-Polarized Orders of Diffraction Journal of Optical Technology 73 (3) 2006: pp. $188-192$.

4. Voronov, D.L., Warwick, T., Padmore, H.A. MultilayerCoated Blazed Grating with Variable Line Spacing and a Variable Blaze Angle Optics Letters $39(21)$ 2014: pp. 6134-6137.

5. Fuh, A.Y.G., Chen, W.K., Cheng, K.T., Liu, Y.C., Liu, C.K., Chen, Y.D. Formation of Holographic Gratings in Polymer-Dispersed Liquid Crystals Using Off-Resonant Light Optical Materials Express 5 (4) 2015: pp. 774-80. https://doi.org/10.1364/OME.5.000774

6. Zharkova, G.M., Petrov, A.P., Samsonova, I.V., Strel'tsov, S.A., Khachaturyan, V.M. Polarization Characteristics of Polymer-Liquid-Crystal Diffraction Gratings Journal of Optical Technology 75 (8) 2008: pp. 514-517.

7. Criante, L., Vita, F., Castagna, R., Lucchetta, D.E., Simoni, F. Characterization of Blue Sensitive Holographic Polymer Dispersed Liquid Crystal for Microholographic Molecular Crystals and Liquid Crystals 465 (1) 2007: pp. 203-215. https://doi.org/10.1080/15421400701205941

8. Jun, H.M., Shu, Y. Creating Three-Dimensional Polymeric Microstructures by Multi-Beam Interference Lithography Journal of Macromolecular Science 45 (4) 2005: pp. 351-373.

9. Liu, Y.J., Sun, X.W. Holographic Polymer-Dispersed Liquid Crystals: Materials, Formation, and Applications Advances in Optoelectronics 2008 (2008) 2008: pp. 684349-1-684349-52.

10. Liu, Y.J., Sun, X.W. Electrically Tunable Two-Dimensional Holographic Photonic Crystal Fabricated by a Single Diffractive Element Applied Physics Letters 89 (17) 2006: pp. 171101-1 171101-3.

11. Matharu, A.S., Jeeva, S., Ramanujam, P.S. Liquid Crystals for Holographic Optical Data Storage Chemical Society Reviews 36 (12) 2007: pp. 1868-1880.

12. Gregory, P.C., James, N.E., Marc, D.R., Andrew, C.J., Robert, A.P. Liquid-Crystal Diffraction Gratings Using Polarization Holography Alignment Techniques Journal of Applied Physics 98 (12) 2005: pp. 123102-1-123102-10.

13. Hsiao, V.K.S., Chang, W.T. Optical Switchable, Polarization-Independent Holographic Polymer Dispersed Liquid Crystal (H-PDLC) Gratings Applied Physics B 100 (3) 2010: pp. 539-546. https://doi.org/10.1007/s00340-010-3939-4

14. Bunning, T.J., Natarajan, L.V., Tondiglia, V.P., Sutherland, R.L., $\quad$ Veziet, D.L., Adams, W.W. The Morphology and Performance of Holographic Transmission Gratings Recorded in Polymer Dispersed Liquid Crystal Polymer 36 (14) 1995: pp. 2699-2708.

15. Liu, J.L., Liu, X.H., Zhen, Z. Effects of Chiral Additives on The Electro-Optical Properties of Polymer Dispersed Liquid Crystal Materials Letters 163 2016: pp. 142-145. 